\title{
Shear test on viscoelastic granular material using Contact Dynamics simula- tions
}

\author{
Juan Carlos Quezada ${ }^{1, \star}$, Loba Sagnol ${ }^{1,2}$, and Cyrille Chazallon ${ }^{1}$ \\ ${ }^{1}$ ICUBE, UMR 7347, CNRS, INSA de Strasbourg, Strasbourg, France \\ ${ }^{2}$ Institut Grund- und Straßenbau, Hochschule Karlsruhe Technik und Wirtschaft, Karlsruhe, Germany
}

\begin{abstract}
By means of 3D contact dynamic simulations, the behavior of a viscoelastic granular material under shear loading is investigated. A viscoelastic fluid phase surrounding the solid particles is simulated by a contact model acting between them. This contact law was implemented in the LMGC90 software, based on the Burgers model. This model is able to simulate also the effect of creep relaxation. To validate the proposed contact model, several direct shear tests were performed, experimentally and numerically using the Leutner device. The numerical samples were created using spheres with two particle size distribution, each one identified for two layers from a road structure. Our results show a reasonable agreement between experimental and numerical data regarding the strain-stress evolution curves and the stress levels measured at failure. The proposed model can be used to simulate the mechanical behavior of multi-layer road structure and to study the influence of traffic on road deformation, cracking and particles pull-out induced by traffic loading.
\end{abstract}

\section{Introduction}

Granular materials are widely used as filling materials since of their mechanical properties such their shear strength and porosity. Examples include construction foundations, earthworks and transport infrastructures such as railway ballast and asphalt concrete.

Asphalt is a complex multi-phase mixture comprises bitumen, graded mineral aggregate and air. This mixture relies on an interlocking the solid phase, conformed by crushed aggregates, for its strength with bitumen to provide a cohesion to the mixture. Herein, the solid phase distributes the forces induced by traffic loading. This material has a typical viscoelastic behavior, where the mechanical performance of the mixture is a function of strain rate and temperature. This macroscopic behavior is mainly dependent of the micro-scale behavior of the asphalt mixture.

Laboratory trials allow to identify the overall response of asphalt mixture, but struggle to obtain a micromechanical insight of these materials. Numerical simulation using continuum mechanics approaches shows the same difficulties regarding the identification of micro-scale properties. A way around this barrier is to use a discrete approach, which has been widely used to model the behavior of granular materials. This method allows to simulate the interaction of a collection of rigid or deformable bodies in contact. Over the past two decades, the discrete element method (DEM) has been used by many researchers to simulate the microstructure of asphalt mixtures. These studies have modeled the asphalt using simple spherical particles,

\footnotetext{
^e-mail: juan-carlos.quezada@insa-strasbourg.fr
}

irregular particles created with clumps of spheres or by generating samples using imaging techniques [1-3].

In this work, a viscoelastic contact law is developed and implemented in a DEM code to model asphalt mixtures. To validate the method and to calibrate the contact model parameters, laboratory shear tests were performed. The initial section (Sect. 2) of this paper presents the experimental setup and laboratory tests. Then, the numerical procedures are outlined (Sect. 3) together with the simulation method, particle properties, and preparation protocol. Finally, in Sect. 4 the results from numerical shear test are analyzed and compared to experimental data to validate the proposed contact model.

\section{Experimental procedures}

\subsection{Experimental setup}

Experimental procedures were performed using the Leutner shear test on asphalt samples. The specimen cores were extracted from an actual double-layer asphalt pavement. This pavement structure test comprises a $60 \mathrm{~mm}$ thick layer for the binder course and a $60 \mathrm{~mm}$ thick layer for the surface course. The particle size distribution of these layers were $0 / 10$ and $0 / 16$ respectively. This pavement structure was constructed on an unbound granular base course. From this asphalt pavement 5 cores were extracted with the following dimensions: $120 \mathrm{~mm}$ height and $150 \mathrm{~mm}$ diameter. 


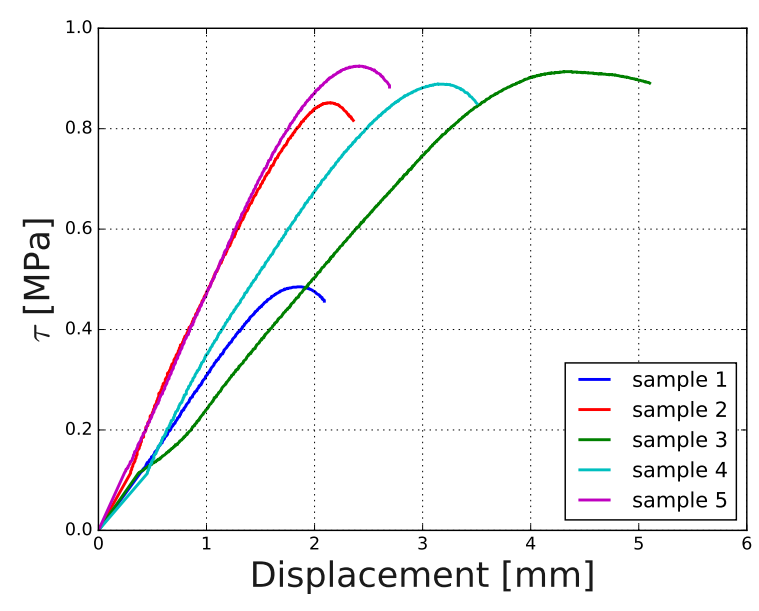

Figure 1. Shear stress-displacement curves obtained for experimental trials.

\subsection{Monotonic shear test}

The shear test were carried-out with the Leutner device [4]. This trial is a destructive monotonic shear test with pure interlayer shearing. During the test the binder course layer of the core was fixed whereas the surface course layer was loaded at a controlled rate of $50 \mathrm{~mm} / \mathrm{min}$ at 25 ${ }^{\circ} \mathrm{C}$ until failure was reached. The equipment records the shear force applied at the interface between the two layers and the displacement of the surface layer.

Figure 1 displays the shear stress $\tau$ as a function of the imposed displacement. The experimental results show a variability in terms of the maximum applied shear stress and measured displacement at failure. From the shear stress-displacement curves, an average value of $0.898 \mathrm{MPa}$ is obtained for the shear-stress and $2.37 \mathrm{~mm}$ for the displacement at failure.

\section{Numerical procedures}

\subsection{Contact dynamics method}

The numerical simulations were performed using the Contact Dynamics (CD) method with spherical particles [57]. The CD method is a discrete element approach for the simulation of non-smooth granular dynamics with contact laws expressing basically the mutual exclusions and dry friction between particles without introducing the elastic or viscous regularization often used in explicit methods such as molecular dynamics [8-11] or the distinct element method [12]. The non-smoothness refers to various degrees of discontinuity in the velocities and contact forces arising in a system composed of rigid particles. In this method, the equations of motion for each particle are formulated as differential inclusions in which velocity jumps replace accelerations $[13,14]$. The unilateral contact interactions and Coulomb friction law are treated as complementarity relations or set-valued contact laws. At each time step, all the velocities and contact forces in the system are determinate simultaneously from the equations of dynamics. This kinematic problem is solved by an iterative procedure based on the non-linear Gauss-Seidel method. For our simulations, we used the LMGC90 software, which is capable of modeling a collection of rigid or deformable particles of various shapes by different algorithms [15].

\subsection{Description of the contact model}

To model an asphalt mixture under a shear loading, a viscoelastic contact law is implemented during the shear test. The chosen contact law was the Burgers model which is a more accurate model of viscoelastic behavior for asphaltic materials [16-18]. It describes finely the creep, relaxation and dynamic properties of asphalt mixtures and has become a commonly used contact model in the DEM simulation of asphalt mixtures. This model consists of the Maxwell model combined in series with the Kelvin-Voigt model. The time dependent normal contact stiffness $K_{n}$ is given by:

$$
K_{n}=\left(\frac{1}{K_{m}}+\frac{t}{\eta_{m}}+\frac{1}{K_{k}}\left(1-e^{-t / t_{r}}\right)\right)
$$

where $K_{m}$ is Maxwell normal stiffness, $\eta_{m}$ is Maxwell normal viscosity, $K_{k}$ is Kelvin-Voigt normal stiffness, $\eta_{k}$ is the Kelvin-Voigt normal viscosity, $t$ is the loading time and $t_{r}=\eta_{k} / K_{k}$ is the relaxation time. The parameter values used in our simulation were $2 \times 10^{6} \mathrm{~N} . \mathrm{m}^{-1}$ for $K_{m}, 2 \times 10^{6}$ N.m ${ }^{-1}$ for $K_{k}, 2 \times 10^{7}$ N.s.m ${ }^{-1}$ and $2 \times 10^{7}$ N.s.m ${ }^{-1}$ for $\eta_{m}$ and $\eta_{k}$ respectively. This set of values generates the best fit regarding the shear stress-displacement curve obtained for the numerical sample in comparison with experimental data. Assuming a perfectly isotropic behavior, tangential stiffness $K_{t}$ and $K_{s}$ are estimated as function of normal stiffness as: $K_{t}=K_{s}=K_{n} /(2(1+v))$ where $v$ is the Poisson's ratio. As a sake of simplicity, this ratio was set to 0.3 .

This contact law was adapted to the CD formulation to be implemented in the LMGC90 software. The equations of dynamics can be written in a compact form by means a matrix representation in the contact frame:

$$
\mathbf{U}^{+}=\mathbf{U}^{-}+\mathbf{W R}
$$

where $\mathbf{U}^{+}$and $\mathbf{U}^{-}$the left-limit and right-limit contact velocities, respectively, $\mathbf{W}$ is the Delassus local matrix and $\mathbf{R}$ the local impulsion at contact. In this form, the accelerations are replaced by velocity jumps defined by $\mathbf{U}^{+}-\mathbf{U}^{-}$ and the equations of motion take the form of an equality between the change of momentum and the average impulse during the time-step $H$. The coefficients $W_{i j}$ from the Delassus local matrix are the inverse reduced inertia. To incorporate our viscoelastic model in the local frame formulation, we added complementary components in this local matrix $W_{i i}^{*}$ for normal and tangential components $(i$ stand for $n, t$ or $s$ ):

$$
W_{i i}^{*}=W_{i i}+1 /\left(H^{2} K_{i}\right)
$$


Table 1. Particle size distribution for each layer composing the sample.

\begin{tabular}{llllll}
\hline diameter(mm) & 2 & 5.6 & 8 & 11.2 & 16 \\
\hline binder course (\%) & 22.9 & 20.1 & 24.0 & 29.8 & 3.2 \\
surface course $(\%)$ & 28.4 & 32.4 & 36.6 & 2.7 & 0.0 \\
\hline
\end{tabular}

To model the brittle behavior of this material, a yield stress criterion is imposed to identify when cohesive contacts are lost. This criterion is verified at each time step, by calculating the generated stress at contact as $K_{i} \times\left(\Delta_{i}\right) / A$, where $K_{i}$ is the stiffness at contact, $\Delta_{i}$ the contact displacement during a time-step and $A$ is the smaller projected surface of particles at contact. When shear or tensile stress reaches the yield value of $2.7 \times 10^{6} \mathrm{~Pa}$, the bond between particles is broken and a frictional contact law is applied instead.

\subsection{Sample preparation}

The numerical samples are composed of spherical rigid particles with two different size distributions. Each particle has a diameter ranging from $2 \mathrm{~mm}$ to $10 \mathrm{~mm}$ for the surface course layer and from $2 \mathrm{~mm}$ to $16 \mathrm{~mm}$ for the binder course layer (see Tab. 1). Here, the graded curve was cut at $2 \mathrm{~mm}$ to avoid modeling all the fines, to reduce simulation time. For each particle size, a specific number of particles are created according to the particle size distribution. Then these particles are disposed randomly within a cubic lattice with $150 \mathrm{~mm}$ diameter and $1.6 \mathrm{~m}$ height. The bulk density for all particles is $2600 \mathrm{~kg} \cdot \mathrm{m}^{-3}$. The coefficient of friction between the particles is set to $\mu=0.7$ which is a typical value used for rock crushed aggregates.

The preparation protocol consists in first pouring the particles of the bottom layer by gravity into a cylindrical box with $150 \mathrm{~mm}$ diameter with zero particle-wall friction. The binder course layer obtained by this procedure is subjected to vibrations of small amplitude $(6 \mathrm{~mm})$ by applying a vertical sinusoidal displacement on the top wall during $1 \mathrm{~s}$ with a frequency of $30 \mathrm{~Hz}$, obtaining a final layer of $60 \mathrm{~mm}$ height. This procedure is repeated for the surface course layer. Finally the resulting sample is confined between two plates inside a cylinder box.

Following this entire process, five samples were prepared. The cylindrical samples have the same geometrical properties as experimental samples: $150 \mathrm{~mm}$ diameter and $120 \mathrm{~mm}$ of total height. The number of particles is about 5200 for all samples, with a packing fraction ranging from 0.584 to 0.59 . Figure 2 shows a snapshot of the resulting numerical sample.

\subsection{Direct shear test modeling}

To model the Leutner shear test, the cylinder box is replaced by two cylinders, each one with $60 \mathrm{~mm}$ height and separated each other of $1 \mathrm{~mm}$. The shear test is performed by imposing a velocity of $50 \mathrm{~mm} / \mathrm{min}$ to the upper cylinder until reach a displacement of $2.5 \mathrm{~mm}$. The shear stress $\tau$

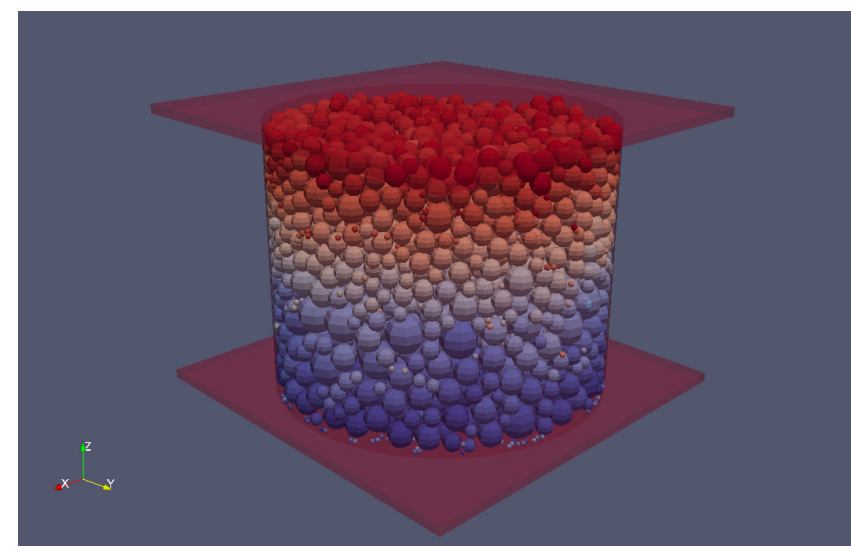

Figure 2. Snapshot of a numerical sample after the preparation stage.

is obtained by measuring the total reaction of contacts between particles and the upper cylinder normalized by the cross section of the sample. The time step was $2 \times 10^{-4} \mathrm{~s}$ in all simulations, and at most 15000 time steps were needed to obtain a displacement of $2.5 \mathrm{~mm}$. The CPU time was $2 \times 10^{-2}$ s per particle and per time step on a Dell computer of speed $3.3 \mathrm{GHz}$.

\section{Validation of the numerical modeling}

\subsection{Shear test results}

Figure 3 depicts different stages during the numerical test. At the beginning, particles from each layer are bonded and stay in equilibrium. When velocity is imposed at the upper cylinder, the upper layer of the sample start to moves as a monolithic assembly, cutting the sample in two halves. The individual velocity of each particle is mapping such as the total reaction between particles and the upper cylinder.

Figure 4 gives the results of the evolution of shear stress $\tau$ as a function of the measured displacement for each numerical sample. For all samples, it is possible to identify a slope at the beginning of the measured displacement until reach failure produced by the shear loading. Once the failure is reached, the system evolves showing a post-peak relaxation. This residual stress value is generated by frictional forces between unbounded particles. The maximum value of shear stress is obtained for a displacement comprised between $2 \mathrm{~mm}$ and $2.3 \mathrm{~mm}$, where this range of values is quite similar to the measured displacement values obtained for the experimental tests.

\subsection{Comparison with experimental results}

In the aim to validate the numerical model, table 2 provides the shear stress and displacement values at failure, for numerical and experimental tests. The average numerical values show a good agreement with experimental data in both shear stress and displacement measured at failure, where the relative error between them is found smaller than $7 \%$. It can be considered as an encouraging result for a validation of the proposed model for the study of asphalt mixtures. 

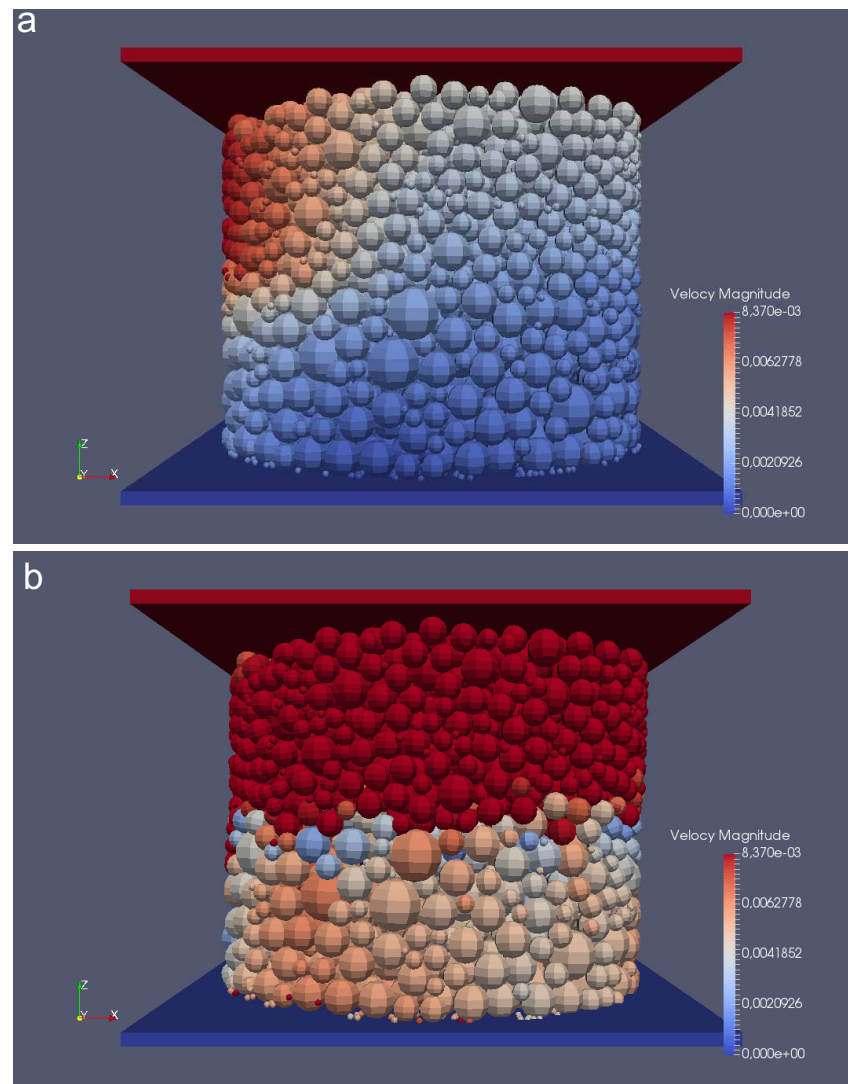

Figure 3. Sketch of average velocity of grains for different stages during the shear test: a) At the beginning of the shear test; b) At failure.

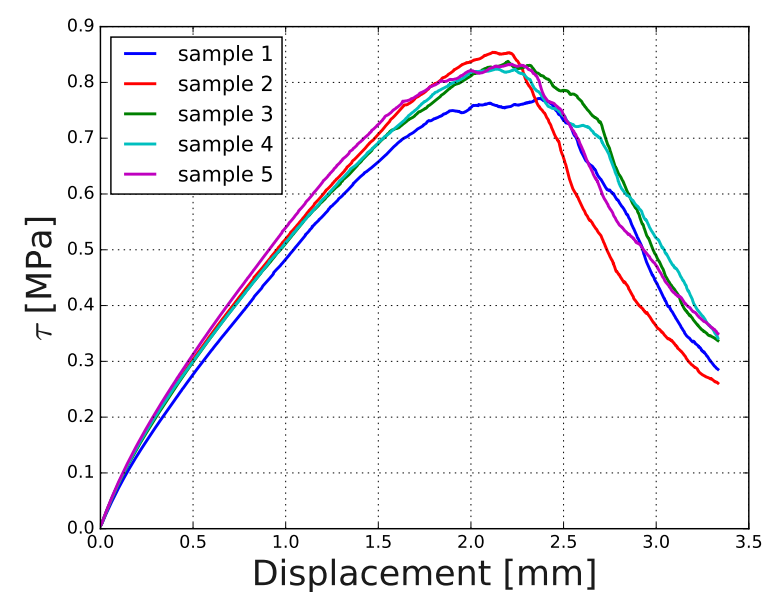

Figure 4. Shear stress $\tau$ evolution as a function of imposed displacement

Table 2. Average values of shear stress $\tau$ and peak displacement $d$ at failure through the experiments and the simulations.

\begin{tabular}{lll}
\hline & $\tau[\mathrm{MPa}]$ & $d[\mathrm{~mm}]$ \\
\hline experiments & 0.898 & 2.37 \\
simulations & 0.837 & 2.2 \\
\hline
\end{tabular}

\section{Concluding remarks}

Our results show that implemented viscoelastic contact model is able to reproduce experimental data from shear test, regarding shear force and maximum displacement at failure. The general results of these simulations for each configuration are in a good agreement with the average values through experiments despite the intrinsic variability within experimental trials. The results of this preliminary work can be considered as encouraging for the validation of the proposed contact model to study the mechanical behavior of multi-layer road structures. The next step in this work will be the inclusion of irregular particles to model actual aggregates to obtain more realistic asphalt mixtures.

\section{References}

[1] A.C. Collop, G.R. McDowell, Y.W. Lee, Granular Matter 8, 175 (2006)

[2] S. Adhikari, Z.P. You, International Journal of Pavement Research and Technology 1, 94 (2008)

[3] W. Cai, G. McDowell, G. Airey, Soils and Foundations 54, 12 (2014)

[4] R. Leutner, Bitumen 41 (1979)

[5] J. Moreau, European Journal of Mechanics A/Solids supp., 93 (1994)

[6] M. Jean, Computer Methods in Applied Mechanic and Engineering 177, 235 (1999)

[7] F. Radjai, V. Richefeu, Mechanics of Materials 41, 715 (2009)

[8] T. Pöschel, V. Buchholtz, J. Phys. I France 5, 1431 (1995)

[9] N.V. Brilliantov, F. Spahn, J.M. Hertzsch, T. Pöschel, Physical review E 53, 5382 (1996)

[10] H. Herrmann, S. Luding, Continuum Mechanics and Thermodynamics 10, 189 (1998)

[11] F. Radjaï, F. Dubois, Discrete-element modeling of granular materials (Wiley-Iste, 2011)

[12] P.A. Cundall, O.D. Strack, Geotechnique 29, 47 (1979)

[13] J.J. Moreau, Unilateral contact and dry friction in finite freedom dynamics, in CISM (1988), pp. 1-82

[14] B. Brogliato, Nonsmooth mechanics (Springer, London, 1999)

[15] F. Dubois, M. Jean, LMGC90, in Actes du sixieme colloque national en calcul des structures (2003), Vol. 1, pp. 111-118

[16] J. Betten, Creep mechanics (Springer Science \& Business Media, 2008)

[17] Y. Liu, Z. You, in Pavements and materials: Modeling, testing, and performance (2009), pp. 26-36

[18] A. Zbiciak, Bulletin of the Polish Academy of Sciences: Technical Sciences 61, 65 (2013) 